\title{
Anisotropic de Gennes Narrowing in Confined Fluids
}

\author{
Kim Nygård, ${ }^{1, *}$ Johan Buitenhuis, ${ }^{2}$ Matias Kagias, ${ }^{3,4}$ Konstantins Jefimovs, ${ }^{3,4}$ Federico Zontone, ${ }^{5}$ and Yuriy Chushkin ${ }^{5}$ \\ ${ }^{1}$ Department of Chemistry and Molecular Biology, University of Gothenburg, SE-41296 Gothenburg, Sweden \\ ${ }^{2}$ Forschungszentrum Jülich, ICS-3, D-52425 Jülich, Germany \\ ${ }^{3}$ Paul Scherrer Institut, CH-5232 Villigen, Switzerland \\ ${ }^{4}$ Institute for Biomedical Engineering, UZH/ETH Zürich, CH-8092 Zürich, Switzerland \\ ${ }^{5}$ European Synchrotron Radiation Facility, 71 Avenue des Martyrs, F-38000 Grenoble, France \\ (Received 19 November 2015; revised manuscript received 16 February 2016; published 21 April 2016)
}

The collective diffusion of dense fluids in spatial confinement is studied by combining high-energy (21 keV) x-ray photon correlation spectroscopy and small-angle x-ray scattering from colloid-filled microfluidic channels. We find the structural relaxation in confinement to be slower compared to the bulk. The collective dynamics is wave vector dependent, akin to the de Gennes narrowing typically observed in bulk fluids. However, in stark contrast to the bulk, the structure factor and de Gennes narrowing in confinement are anisotropic. These experimental observations are essential in order to develop a microscopic theoretical description of collective diffusion of dense fluids in confined geometries.

DOI: 10.1103/PhysRevLett.116.167801

Dense fluids in narrow spatial confinement exhibit complex microscopic ordering, due to competing packing constraints imposed by the confining surfaces and the other particles in the system [1]. As a result confinement alters the fluids' dynamic properties [2], and is thus highly relevant in a wide range of scientific phenomena and technological applications, including water transport in molecular sieves, the hindered motion of colloidal dispersions in porous matrices, the glass transition in thin films, lubrication, and various micro- and nanofluidic applications. State-of-the-art experiments [3] and simulations [4] have shown that packing constraints in confined geometries lead to position-dependent diffusion of dense fluids, thereby highlighting the close connection between microscopic structure and dynamics. Correlations between structural quantities such as the excess entropy, on the one hand, and dynamic properties such as singleparticle diffusion [5] or structural relaxation [6], on the other hand, have also been reported. However, nanoscopically confined dense fluids exhibit complex dynamical behavior [7], and a conceptually simple mechanistic picture in terms of the microscopic structure is still missing.

An established description of microscopic dynamics in the bulk is provided via a phenomenon known as de Gennes narrowing [8], according to which the wave vector dependent collective diffusion coefficient $D(\mathbf{q})$ scales as the inverse of the structure factor $S(\mathbf{q})$. In essence, density fluctuations with a wave vector $\mathbf{q}$ corresponding to a

Published by the American Physical Society under the terms of the Creative Commons Attribution 3.0 License. Further distribution of this work must maintain attribution to the author(s) and the published article's title, journal citation, and DOI. maximum in the structure factor have a low free energy cost and thus decay slowly, providing a straightforward physical connection between microscopic structure and dynamics. This scheme has been used to analyze collective diffusion in bulk systems ranging from colloidal dispersions [9] to glass-forming silicates [10], and has even been successfully applied to the relative motion of protein domains [11]. However, its validity in confinement is yet to be demonstrated, whether experimentally, theoretically, or by simulations. Spatial confinement induces anisotropy in the fluid's pair correlations [12] and the ensuing structure factor [13]. If de Gennes narrowing holds in confinement, then one would observe anisotropic wave vector dependent collective diffusion.

In this Letter, we study the connection between the microscopic structure and dynamics of dense fluids confined between planar walls at close separation, i.e., in a narrow slit geometry. We have recently developed a unique scheme to experimentally determine anisotropic structure factors of confined fluids, based on small-angle x-ray scattering (SAXS) from colloid-filled micro- or nanofluidic channel arrays [14], in semiquantitative agreement with $a b$ initio theoretical predictions $[15,16]$. Here, we extend the methodology for simultaneous determination of the static structure factor and the wave vector dependent collective diffusion coefficient of a charge-stabilized colloidal dispersion in narrow confinement, by carrying out high-energy SAXS and $\mathrm{x}$-ray photon correlation spectroscopy (XPCS) experiments. We provide the first observation of anisotropic de Gennes narrowing in confined fluids, with the structural relaxation being highly anisotropic and significantly slower compared to the bulk. These experimental findings, which establish a connection between structure and dynamics at the fundamental level of 
pair densities, are crucial for the development of a microscopic theory of dynamics in dense confined fluids.

Following Refs. [14-16], we used a colloidal dispersion confined in a specifically designed microfluidic channel array as a model for confined fluids. The colloid consisted of charge-stabilized spherical silica particles dispersed in ethylene glycol, with a particle volume fraction of $\phi=0.168$ in the bulk. The average diameter of the particles was $\sigma=182 \mathrm{~nm}$ and the polydispersity $\Delta \sigma / \sigma=1.5 \%$, as determined by SAXS from a dilute bulk dispersion. Details on the synthesis of the colloidal dispersion can be found elsewhere [17].

The microfluidic container, in turn, consisted of a periodic array of one-dimensional channels. The channel array was made into a $300 \mu \mathrm{m}$ thick silicon wafer by electron-beam lithography and $\mathrm{KOH}$ etching following Ref. [18]. It had a period of $2 \mu \mathrm{m}$, a depth of $\approx 18 \mu \mathrm{m}$, and a channel width of $H=490 \mathrm{~nm}$ (i.e., $H \approx 2.7 \sigma$ ), as determined by scanning electron microscopy. The resulting confining walls were structureless on the length scales relevant for the colloidal dispersion, facilitating studies on the connection between microscopic structure and dynamics. We also prepared space for a bulk fluid reservoir, allowing us to collect bulk data from the same sample cell. Finally, we covered the channel array by a $500 \mu \mathrm{m}$ thick glass plate in order to prevent evaporation of the solvent and to facilitate attachment of syringes for filling the fluid, resulting in a $3 \mu \mathrm{m}$ thin fluid film between the channel array and the glass cover.

We carried out the combined XPCS and SAXS experiment at beam line ID10 of the European Synchrotron Radiation Facility (ESRF). Our key technical advance is the use of high-energy incident $x$ rays, thereby minimizing absorption in the beam path and allowing the XPCS experiment on the colloidal dispersion sandwiched between the silicon wafer and the glass cover. The incident $\mathrm{x}$-ray beam had an energy of $\hbar \omega=21 \mathrm{keV}$, and a size of $10 \times 10 \mu \mathrm{m}^{2}$ at the sample position, and impinged parallel to the confining walls. To maximize the intensity the $\mathrm{x}$-ray beam was focused onto the sample plane and an evacuated flight tube was placed between the sample and the detector to minimize the background from air scattering. Scattered $\mathrm{x}$ rays were collected in transmission mode $5.3 \mathrm{~m}$ behind the sample in a twofold manner as follows. First, we obtained an overview of the static structure using the two-dimensional single-photoncounting CdTe MAXIPIX detector $(256 \times 768$ pixels with a size of $55 \times 55 \mu \mathrm{m}^{2}$ each) [19]. Then, we collected static SAXS and dynamic XPCS data simultaneously, in selected scattering vector directions with respect to the confining channels, using a $0.1 \times 0.1 \mathrm{~mm}^{2}$ slit opening in front of a point detector, a Cyberstar scintillation counter connected to a Flex hardware correlator. The experiment was carried out at room temperature.

First, we discuss the static SAXS intensity $I(\mathbf{q})$ obtained from the confined fluid, shown in Fig. 1(a). These data, like all data presented in this Letter, have been verified in several repeat measurements. The data are proportional to the anisotropic structure factor [21], $\quad S(\mathbf{q})=1+$ $\frac{1}{N} \iint n(\mathbf{r}) n\left(\mathbf{r}^{\prime}\right) h\left(\mathbf{r}, \mathbf{r}^{\prime}\right) e^{i \mathbf{q} \cdot\left(\mathbf{r}-\mathbf{r}^{\prime}\right)} d \mathbf{r} d \mathbf{r}^{\prime}$, where $\mathbf{q}$ denotes the wave or scattering vector, $N$ is the total number of particles, $n(\mathbf{r})$ is the number density profile across the confining slit, $h\left(\mathbf{r}, \mathbf{r}^{\prime}\right)$ is the pair correlation function, and the integration is carried out over particle positions $\mathbf{r}$ and $\mathbf{r}^{\prime}$. For the present case of a fluid in slit confinement, $S(\mathbf{q})$ is essentially given by the Fourier transform of the pair density correlation $n(\mathbf{r}) h\left(\mathbf{r}, \mathbf{r}^{\prime}\right)$, ensemble averaged over particle positions across the confining channel (for illustrative real-space representations, see Ref. [13]). In stark contrast to isotropic bulk fluids, the SAXS intensity obtained from the confined fluid is strongly anisotropic, directly demonstrating the strong anisotropy in the structure factor and the underlying pair correlations.

Structural relaxation in the fluid was probed by XPCS, which measures the intensity autocorrelation function

$$
g_{2}(\mathbf{q}, t)=\frac{\left\langle I\left(\mathbf{q}, t_{0}\right) I\left(\mathbf{q}, t_{0}+t\right)\right\rangle_{t_{0}}}{\left\langle I\left(\mathbf{q}, t_{0}\right)\right\rangle_{t_{0}}\left\langle I\left(\mathbf{q}, t_{0}+t\right)\right\rangle_{t_{0}}},
$$

where $\langle\cdots\rangle_{t_{0}}$ denotes a temporal average over all times $t_{0}$ [22]. The $g_{2}(\mathbf{q}, t)$ curves obtained from both the confined and the bulk fluid at a scattering vector magnitude of $q=0.034 \mathrm{~nm}^{-1}$ are presented in Fig. 1(b), the former in the nonparallel direction as defined in Fig. 1(a). These correlation functions differ in two ways. First, the dynamics is significantly slower in confinement compared to the bulk at this particular scattering vector. Second, the shape of the correlation function in confinement is stretched, in contrast to the bulk.

The intensity autocorrelation functions of Fig. 1(b) are related to the fluids' density fluctuations via the Siegert relation $g_{2}(\mathbf{q}, t)=1+\gamma|f(\mathbf{q}, t)|^{2}$, where $\gamma$ denotes the experimental contrast and $f(\mathbf{q}, t)$ denotes the normalized intermediate scattering function [22]. For quantitative analysis we fit the correlation functions with the Siegert relation using the phenomenological Kohlrausch-WilliamsWatts exponential expression $f(\mathbf{q}, t)=\exp \left\{-[t / \tau(\mathbf{q})]^{\beta}\right\}$, which is commonly applied to describe dynamics in disordered systems. Here, $\tau(\mathbf{q})$ denotes the wave vector dependent relaxation time and $0<\beta \leq 2$ denotes the shape factor with $\beta<1$ corresponding to the stretched correlation function. From the fits of the $g_{2}(\mathbf{q}, t)$ curves, we obtain a nearly constant $\beta \approx 0.95$ for the bulk, $\approx 0.88$ for the fluid film, as well as $\approx 0.72, \approx 0.70$, and $\approx 0.78$ for the confined fluid in the diagonal, nonparallel, and parallel directions [see Fig. 1(a) for definitions]. To account for the stretching we consider throughout this study an average relaxation time $\langle\tau(\mathbf{q})\rangle \equiv \int_{0}^{\infty} f(\mathbf{q}, t) d t=\tau(\mathbf{q}) \beta^{-1} \Gamma^{*}\left(\beta^{-1}\right)$, where $\Gamma^{*}$ denotes the gamma function.

Stretching of the correlation function indicates a distribution of relaxation times coming either from the sample 

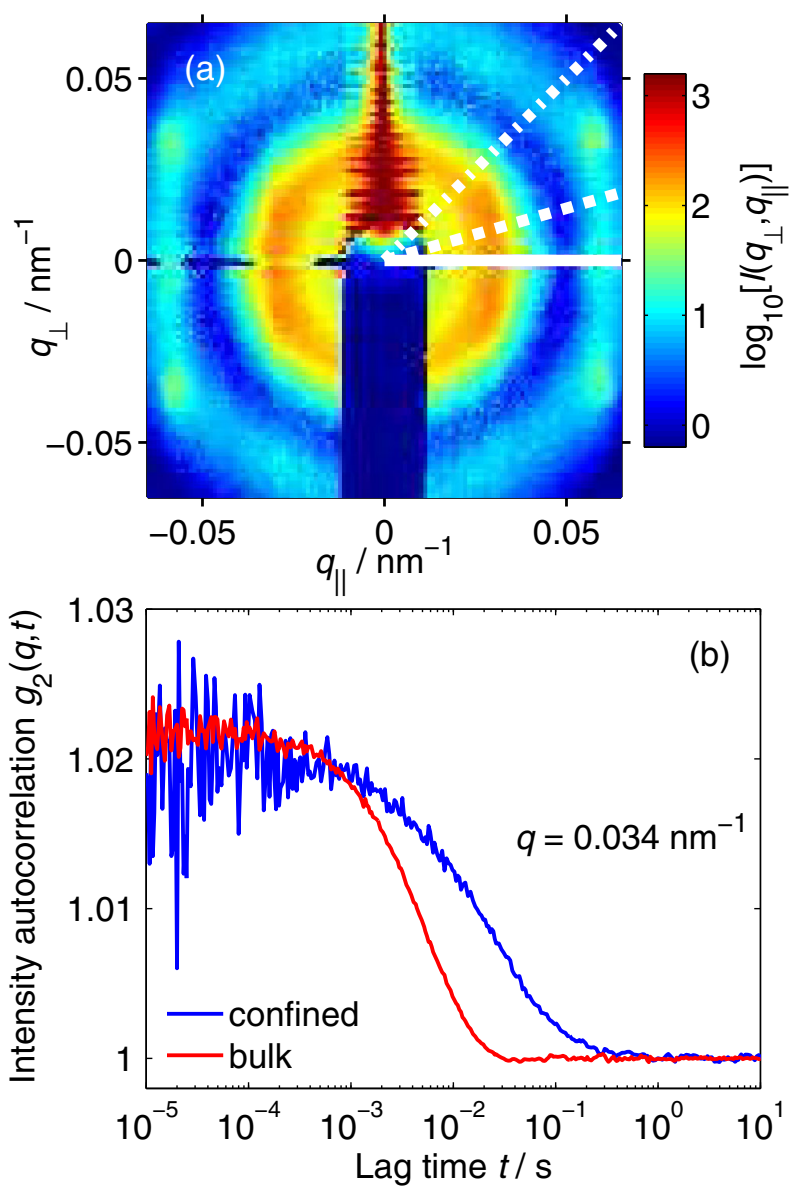

FIG. 1. (a) Static SAXS intensity $I(\mathbf{q})$ obtained from the confined fluid, presented on a log scale as a function of scattering vector components parallel $\left(q_{\|}\right)$and perpendicular $\left(q_{\perp}\right)$ to the confining channels. The solid, dashed, and dashed-dotted white lines depict the parallel $\left(0^{\circ}\right)$, nonparallel $\left(16^{\circ}\right)$, and diagonal $\left(45^{\circ}\right)$ directions (with respect to the confining channels) in which we collect dynamic data. The dark red and blue features at $q_{\|}=0$ are due to diffraction from the confining container and the central beam stop, respectively, and should be neglected in the discussion [20]. (b) Intensity autocorrelation functions $g_{2}(q, t)$ collected from the confined (blue) and bulk (red) colloidal dispersion at a scattering vector magnitude of $q=0.034 \mathrm{~nm}^{-1}$. The data from the confined fluid are collected at an angle of $16^{\circ}$ with respect to the confining channels [denoted nonparallel; see the dashed line of (a) for a graphical definition].

itself or as a result of the experimental conditions. Because our sample contains monodisperse particles, we attribute the weak stretching in the bulk $(\beta \approx 0.95)$ to the averaging of relaxation times over a large slit opening $\left(0.1 \times 0.1 \mathrm{~mm}^{2}\right)$ in front of the point detector. The data from the confined fluid ( $18 \mu \mathrm{m}$ thick) also contain a contribution from the $3 \mu \mathrm{m}$ thick fluid film on top of the channel array [23], which could explain the stronger stretching $(\beta \approx 0.75)$ compared to the bulk. However, the enhanced stretching observed in the film $(\beta \approx 0.88)$ indicates that confinement indeed induces stretched dynamics in our fluid. Our present experimental design does not allow XPCS experiments on the confined fluid alone, and in the future a new cell geometry will be envisaged to study this effect. Importantly, the contribution from the fluid film does not depend on the azimuthal angle, and cannot therefore explain the main observation in our studyanisotropic de Gennes narrowing.

Having introduced the static structure factor $S(\mathbf{q})$ and the wave vector dependent relaxation time $\tau(\mathbf{q})$, we can now address the main question of the present study-how does the microscopic structure of the confined fluid affect its collective diffusion? For this purpose we present in Fig. 2(a) the structure factor $S(\mathbf{q})$, as obtained by dividing the SAXS intensity $I(\mathbf{q})$ by the form factor of a dilute dispersion. The data are shown for four different cases: the confined fluid in the diagonal, nonparallel, and parallel directions as well as the bulk fluid. We note two interesting features in the data. First, the peak positions and intensities
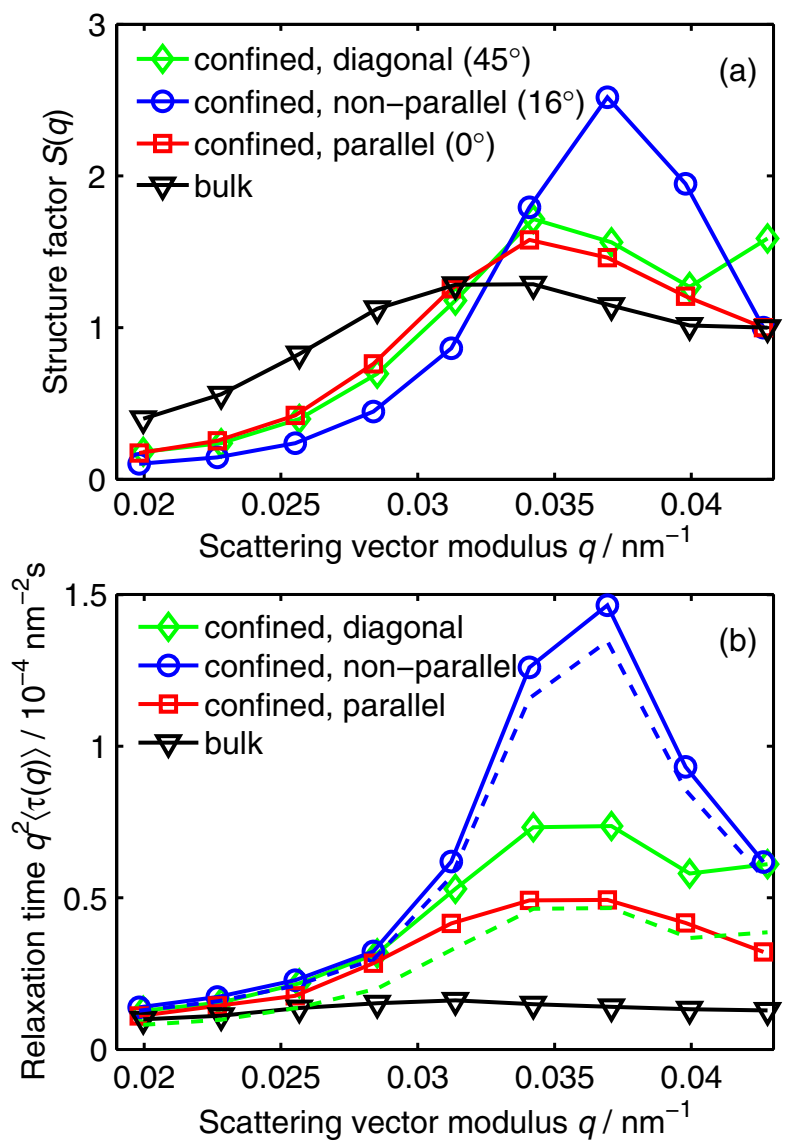

FIG. 2. (a) Static structure factor $S(\mathbf{q})$ obtained from the static SAXS data. (b) Reduced wave vector dependent relaxation time $q^{2}\langle\tau(\mathbf{q})\rangle$, as determined from the dynamic XPCS data using the Kohlrausch-Williams-Watts model (see the text for details). Data are shown for the confined fluid in the diagonal (green diamonds), nonparallel (blue circles), and parallel (red squares) directions as well as for the bulk fluid (black triangles). The dashed lines correspond to reduced relaxation times for the confined fluid, normalized to the effective viscosity of the solvent in the parallel direction (see the text for details). 
differ between the bulk and confinement, implying a denser packing of particles in the latter case. A possible explanation may be the formation of dense particle monolayers near each confining wall, which has been observed previously for charge-stabilized colloids in confinement [24]. Second, the data for the confined fluid are direction dependent, as inferred already from the anisotropic SAXS data in Fig. 1(a), demonstrating an anisotropic $S(\mathbf{q})$ and implying the ensuing anisotropic properties of the confined fluid. Such an anisotropy in $S(\mathbf{q})$ has previously been observed for both charged [14] and hard-sphere colloids $[15,16]$ in spatial confinement.

The wave vector dependent decay rate of density fluctuations, $\Gamma(\mathbf{q})=1 / \tau(\mathbf{q})$, can be cast in the form of the collective diffusion coefficient, $\Gamma(\mathbf{q})=q^{2} D(\mathbf{q})$. On the other hand, de Gennes narrowing implies that the latter behaves as the inverse of the structure factor, $D(\mathbf{q}) \propto 1 / S(\mathbf{q})$. We thus expect the reduced wave vector dependent relaxation time to be proportional to the structure factor, $q^{2} \tau(\mathbf{q}) \propto S(\mathbf{q})$ [22]. This behavior is indeed observed in Fig. 2(b), where we present $q^{2}\langle\tau(\mathbf{q})\rangle$ for the same four systems as in Fig. 2(a); both for the bulk and confinement we observe a maximum in the average reduced relaxation time $q^{2}\langle\tau(\mathbf{q})\rangle$ coinciding with the maximum in the structure factor $S(\mathbf{q})$. This contrasts with the constant reduced Stokes-Einstein relaxation time observed for dilute fluids, $q^{2} \tau=6 \pi \eta r_{H} / k_{B} T$ with $k_{B}$ Boltzmann's constant, $T$ the absolute temperature, $r_{H}$ the particles' hydrodynamic radius $(\approx 91 \mathrm{~nm}$ in this study), and $\eta$ the solvent's viscosity [22]. Most importantly, the anisotropic $S(\mathbf{q})$ for the confined fluid leads to an anisotropic $q^{2}\langle\tau(\mathbf{q})\rangle$, with both functions exhibiting a similar behavior as a function of wave vector direction. We emphasize that the slower dynamics in the nonparallel direction compared to the diagonal direction can only be explained by anisotropic caging effects due to neighboring particles. To the best of our knowledge, this is the first observation of anisotropic de Gennes narrowing in confined fluids, highlighting the importance of describing dense confined fluids at the level of anisotropic pair densities.

The agreement between $q^{2}\langle\tau(\mathbf{q})\rangle$ and $S(\mathbf{q})$ in Fig. 2 is only qualitative. This is notable when comparing data collected in the parallel and diagonal directions; the static structure factors are similar, but the dynamics is slower in the latter case. A possible explanation is that colloidal dispersions also exhibit hydrodynamic interactions mediated by the solvent. In particular, we expect a viscous drag effect due to the presence of a solid surface, which depends on both the direction and the particle's distance from the wall (see, e.g., Ref. [25]). To estimate the magnitude of this effect, we have determined effective $q$-independent viscosities in the diagonal, nonparallel, and parallel directions following Ref. [25], assuming a uniform distribution of particle distances from the walls and employing the superposition approximation of Ref. [26]. In Fig. 2(b) we show as dashed lines the reduced relaxation times in the diagonal and nonparallel directions, normalized to the lower effective viscosity parallel to the confining walls. Although this normalization procedure is only approximate, it is clear that the quantitative differences in $q^{2}\langle\tau(\mathbf{q})\rangle$ between the diagonal and parallel directions can (at least partly) be attributed to the viscous drag effect. Nevertheless, the strongest slowing down in the nonparallel $\left(16^{\circ}\right)$ direction as compared to the diagonal $\left(45^{\circ}\right)$ and parallel $\left(0^{\circ}\right)$ direction demonstrates that a higher structure factor peak seems the strongest predictor for slower dynamics, i.e., de Gennes narrowing.

In principle our experimental results could be analyzed using mode-coupling theory, which is a dynamic counterpart of the theoretical approach used in Refs. [15,16]. However, although mode-coupling theory has recently been extended to confined hard-sphere fluids, the calculations have remained difficult and scarce in practice [7,27]. Moreover, a quantitative analysis of the data in Fig. 2(b) would require the inclusion of both hydrodynamic particleparticle [9] and particle-wall [25] interactions, which is highly nontrivial for the present case of a dense fluid in narrow confinement. Such an analysis, which may include the counterbalancing of hydrodynamic particle-particle and particle-wall interactions [28], has not yet been worked out theoretically for dense confined fluids and is beyond the scope of the present study. It is our hope that the anisotropic de Gennes narrowing reported here will both guide theoretical development and facilitate the interpretation of experiments on structural relaxation in dense confined fluids.

Finally, we comment on a promising future application of the methodology exploited here. Recent Monte Carlo simulations and calculations within mode-coupling theory have predicted the existence of reentrant glass transitions in confined hard-sphere systems upon varying the surface separation $H$ [27]. Our approach provides a means to experimentally verify this intriguing prediction; by carrying out a combined SAXS and XPCS experiment for dense hard-sphere fluids as a function of $H$, one could determine how the onset of the glass transition depends on the confining surface separation.

In summary, we have simultaneously probed the microscopic structure and wave vector dependent collective diffusion of spatially confined fluids, by combining high-energy SAXS and XPCS experiments on a colloidal dispersion confined in specifically designed microfluidic channel arrays. Most importantly, we report the first observation of anisotropic de Gennes narrowing in confined fluids, with the anisotropic structure factor showing up as anisotropic wave vector dependent collective diffusion. Our results establish a direct connection between the structure and dynamics in confined fluids at the fundamental level of anisotropic pair densities, and thereby 
provide an important conceptual step towards a microscopic description of collective diffusion in dense confined fluids.

We thank Marie Ruat for the help with the CdTe MAXIPIX detector and the ESRF for providing beam time. K. N. acknowledges the Swedish Research Council for financial support.

*kim.nygard@chem.gu.se

[1] Fundamentals of Inhomogeneous Fluids, edited by D. Henderson (Marcel Dekker, New York, 1992).

[2] S. Granick, Science 253, 1374 (1991).

[3] C. R. Nugent, K. V. Edmond, H. N. Patel, and E. R. Weeks, Phys. Rev. Lett. 99, 025702 (2007).

[4] J. Mittal, T. M. Truskett, J. R. Errington, and G. Hummer, Phys. Rev. Lett. 100, 145901 (2008).

[5] J. Mittal, J. R. Errington, and T. M. Truskett, Phys. Rev. Lett. 96, 177804 (2006).

[6] T. S. Ingebrigtsen, J. R. Errington, T. M. Truskett, and J. C. Dyre, Phys. Rev. Lett. 111, 235901 (2013).

[7] S. Lang, V. Boţan, M. Oettel, D. Hajnal, T. Franosch, and R. Schilling, Phys. Rev. Lett. 105, 125701 (2010).

[8] P. G. de Gennes, Physica (Amsterdam) 25A, 825 (1959).

[9] A. J. Banchio, J. Gapinski, A. Patkowski, W. Häußler, A. Fluerasu, S. Sacanna, P. Holmqvist, G. Meier, M. P. Lettinga, and G. Nägele, Phys. Rev. Lett. 96, 138303 (2006).

[10] B. Ruta, G. Baldi, Y. Chushkin, B. Rufflé, L. Cristofolini, A. Fontana, M. Zanatta, and F. Nazzani, Nat. Commun. 5, 3939 (2014).

[11] L. Hong, N. Smolin, and J. C. Smith, Phys. Rev. Lett. 112, 158102 (2014).

[12] R. Kjellander and S. Sarman, J. Chem. Soc., Faraday Trans. 87, 1869 (1991).
[13] K. Nygård, S. Sarman, and R. Kjellander, J. Chem. Phys. 139, 164701 (2013).

[14] K. Nygård, D. K. Satapathy, J. Buitenhuis, E. Perret, O. Bunk, C. David, and J. F. van der Veen, Europhys. Lett. 86, 66001 (2009).

[15] K. Nygård, R. Kjellander, S. Sarman, S. Chodankar, E. Perret, J. Buitenhuis, and J. F. van der Veen, Phys. Rev. Lett. 108, 037802 (2012).

[16] K. Nygård, S. Sarman, K. Hyltegren, S. Chodankar, E. Perret, J. Buitenhuis, J. F. van der Veen, and R. Kjellander, Phys. Rev. X 6, 011014 (2016).

[17] J. Gapinski, A. Patkowski, A. J. Banchio, J. Buitenhuis, P. Holmqvist, M. P. Lettinga, G. Meier, and G. Nägele, J. Chem. Phys. 130, 084503 (2009).

[18] P. Mao and J. Han, Lab Chip 9, 586 (2009).

[19] C. Ponchut, J. M. Rigal, J. Clement, E. Papillon, A. Homs, and S. Petitdemange, J. Instrum. 6, C01069 (2011).

[20] Because of diffraction from the grating, we cannot measure $S(\mathbf{q})$ and $D(\mathbf{q})$ at angles close to $90^{\circ}$ with respect to the confining channels.

[21] Following Refs. [14,15], we have carefully subtracted scattering data from the fluid film between the channel array and the glass cover.

[22] G. Grübel and F. Zontone, J. Alloys Compd. 362, 3 (2004).

[23] The scattering volume of the confined fluid is $\approx 50 \%$ larger than that of the fluid film.

[24] D. K. Satapathy, O. Bunk, K. Jefimovs, K. Nygård, H. Guo, A. Diaz, E. Perret, F. Pfeiffer, C. David, G. H. Wegdam et al., Phys. Rev. Lett. 101, 136103 (2008).

[25] P. Holmqvist, J. K. G. Dhont, and P. R. Lang, J. Chem. Phys. 126, 044707 (2007).

[26] B. Lin, J. Yu, and S. A. Rice, Phys. Rev. E 62, 3909 (2000).

[27] S. Mandal, S. Lang, M. Gross, M. Oettel, D. Raabe, T. Franosch, and F. Varnik, Nat. Commun. 5, 4435 (2014).

[28] V. N. Michailidou, G. Petekidis, J. W. Swan, and J. F. Brady, Phys. Rev. Lett. 102, 068302 (2009). 